\title{
Immunohistochemical Characterization of Insulin, Glucagon, PDX1, SOX17 and NGN3 Expression in Human Fetal Pancreatic Development
}

\section{Sarah J Anderson', Karen L Seeberger', Cara E Ellis' ${ }^{1}$, Alana Eshpeter ${ }^{1}$ and Gregory S Korbutt ${ }^{1,2,3 *}$}

${ }^{1}$ Alberta Diabetes Institute and University of Alberta, Edmonton, Alberta, T6G 2E1, Canada

${ }^{2}$ Department of Surgery, University of Alberta, Edmonton, Alberta, T6G 2E1, Canada

${ }^{3}$ Alberta Diabetes Institute, 5-002 Li Ka Shing Center, University of Alberta, Edmonton, Alberta, T6G 2E1, Canada

\begin{abstract}
Examination of human fetal pancreatic endocrine cell development can provide further insight in, defining the developmental patterns of endocrine cells and identifying $\beta$-cell progenitors. In this study we performed a comprehensive immunohistochemical analysis of human fetal pancreatic sections aged from 7.7 to 38 weeks post conception (wpc), as well as 10 weeks post natal (wpn), and adult sections. We examined expression and co-expression of insulin, glucagon, cytokeratin19 (CK19), vimentin as well as the transcription factors PDX1, SOX17 and NGN3. Insulin and glucagon expression significantly increased in the first (1-12 wpc) and second (13-24 wpc) trimesters and formed islet-like clusters which resembled adult human islets in the third $(24-38 \mathrm{wpc})$ trimester. Insulin and glucagon coexpressing cells were observed from 8.4 to $23 \mathrm{wpc}$ and peaked during the first trimester. PDX1 expression was observed predominantly in duct-like structures prior to $15 \mathrm{wpc}$, then, was localized to islet structures in the second trimester at 17 wpc. Co-localization of PDX1 and insulin was observed throughout fetal development and in most insulin cells. SOX17 expressing cells were in spatial proximity to the glucagon expressing cells late in the first and second trimesters and did not co-express either insulin or glucagon. NGN3 was detected from 7.7 to 14.4 wpc within the pancreatic mesenchyme. Expression peaked between 10.6-12.1 wpc and was not detected past $15 \mathrm{wpc}$. NGN3 cells co-expressed vimentin but did not co-express insulin or CK19. We present a unique qualitative assessment of insulin, glucagon, PDX1, SOX17 and NGN3 expression and co-expression patterns, during human fetal pancreatic development. In combination with in vitro human embryonic stem cell isolation studies, in vivo characterization of $\beta$-cell progenitors during fetal development, will improve progenitor cell isolation and differentiation of viable $\beta$-cell progenitors destined for transplantation.
\end{abstract}

Keywords: Development; Fetal; Pancreatic; Insulin; Glucagon; Immunofluorescence

Abbreviations: WPC: Weeks Post-conception; WPN: Weeks Post-natal; PDX1: Pancreatic Duodenal Homeobox 1; SOX17: Sex Determining Region Y Box-17; NGN3: Neurogenin3

\section{Introduction}

Understanding the normal developmental biology associated with pancreatic endocrine cell ontogeny is key to identifying $\beta$-cell progenitors and refining in vitro differentiation of these stem cells. In transgenic mouse models [1-3], several differences between human and rodent islet biology have been noted [4] including; the timing of developmental stages [5], the sequence of key developmental events [6,7], composition and organization of islet cell clusters [8-10] including a lower proportion of $\beta$-cells in human islets compared to mouse islets [10], nerve innervation [11] and insulin gene number (2 in mice and 1 in humans) [12]. Therefore, examination of human fetal pancreatic endocrine cell development can provide further insight in defining the developmental patterns of endocrine cells.

Studies examining the human fetal pancreas have used both immunohistochemistry and gene expression to observe changes in islet morphology and to examine pancreatic ontogeny $[4,13,14]$. Unlike mouse development this does not include a late gestation secondary transition islet formation $[7,15]$, rather islets are observed at the end of the first trimester $[13,15]$. Studies conducted by Bouwens et al. [16], Polak et al. [17], and Meier et al. [18] determined that endocrine hormone expressing cells first appear within or adjacent to primitive ductal epithelium suggesting that islet cells differentiate from progenitor cells located in this region. In addition, primitive endocrine cells that co-express insulin and glucagon in early fetal development later mature into mono-hormonal cells Polak et al. [17] and Piper et al. [5]. Recently Riedel et al. [19] suggested that these co-expressing insulin and glucagon cells give rise to mature $\alpha$-cells, since these cells also produce the $\alpha$-cell transcription factor, aristaless related homeobox (ARX) and lack specific $\beta$-cell transcription factors. Pancreatic duodenal homeobox factor-1 (PDX1) is a key transcription factor necessary in pancreatic development and is initially expressed in pancreatic progenitor cells and is later restricted to mature $\beta$-cells [20]. Piper et al. [5] and Lyttle et al. [21] found that cells expressing PDX1 are, initially located within the primitive ductal epithelium and later transition to the islet. Sex-determining region Y box 17 (SOX17) is a transcription factor with a functional role in lineage specification and is expressed early in endoderm development [22-26] and gastrointestinal endoderm [22,27] including the pancreas [24-27]. The transcription factor Neurogenin3 (NGN3) belonging to the basic helix-loophelix (bHLH) class of transcription factors is crucial for mammalian

*Corresponding author: Dr. Gregory S. Korbutt, Alberta Diabetes Institute, 5-002 Li Ka Shing Center, University of Alberta, Edmonton, Alberta, T6G 2E1, Canada, Tel: (780) 492-4657; Fax: (780) 492-5501; E-mail:korbutt@ualberta.ca

Received June 21, 2013; Accepted September 12, 2013; Published September 26, 2013

Citation: Anderson SJ, Seeberger KL, Ellis CE, Eshpetzer A, Korbutt GS (2013) Immunohistochemical Characterization of Insulin, Glucagon, PDX1, SOX17 and NGN3 Expression in Human Fetal Pancreatic Development. J Stem Cell Res Ther 3: 148. doi:10.4172/2157-7633.1000148

Copyright: $(2013$ Anderson SJ, et al. This is an open-access article distributed under the terms of the Creative Commons Attribution License, which permits unrestricted use, distribution, and reproduction in any medium, provided the original author and source are credited. 
endocrine pancreas development [28-32]. It is expressed early in pancreatic progenitor cells and regulates other transcription factor expression in endocrine progenitor cells. Mouse knock-out studies of NGN3 [28-30] have demonstrated that mice deficient for NGN3 fail to develop islets and lack all endocrine cells.

In this study we performed immunohistochemical analysis of human fetal pancreatic sections aged from 7.7 to 38 weeks post conception (wpc), as well as 10 weeks post natal (wpn), and adult sections. We examined the expression of insulin, glucagon, cytokeratin19 (CK19), vimentin as well as the transcription factors PDX1, SOX17 and NGN3. The purpose of this study was to examine the expression and co-expression relationships of these factors, and the changing islet morphology throughout human fetal development. This study confirms previous findings [22-24] and presents new observations of the transcription factor SOX17 as well as NGN3 during human endocrine pancreas development. A detailed knowledge of developing islet morphology, maturation of $\beta$-cells, and expression of key hormones and transcription factors may aid in differentiating functional $\beta$-cells in therapeutic numbers for islet transplantation.

\section{Materials and Methods}

\section{Human sections}

Human fetal pancreatic tissue sections ranging in age from 7.7 to 14.4 weeks post conception (wpc) were obtained from the Birth Defects Research Laboratory at the University of Washington in compliance with US State and Federal regulations. Specimens were collected within $12 \mathrm{~h}$ post-mortem, and fixed with formalin (Richard-Allan Scientific, Kalamazoo, MI, USA). Human fetal pancreatic tissue sections ranging in age from 15 weeks to $38 \mathrm{wpc}$ were also obtained from Alberta Health Services. The 10 weeks post natal (wpn) sample was obtained from the Alberta Diabetes Institute (ADI) IsletCore. The adult pancreatic samples were obtained from the Edmonton Clinical Islet Transplant Program and protocols were approved by the $U$ of A Research Ethics Office. All samples were embedded in paraffin, cut into 5 um sections, and mounted on histobond slides (Fischer Scientific, Ontario, and Canada).

\section{Immunofluorescent staining}

Paraffin sections were rehydrated, and immersed in Tris EDTA for antigen retrieval in a Sanyo Model-F301 1260W microwave for 15 minutes at $80 \%$, and let cool for 20-25 minutes. Sections were rinsed with phosphate buffered saline (PBS), and blocked with $20 \%$ normal goat serum (NGS) in PBS for $1 \mathrm{~h}$. If biotinylated primary antibodies were used, and avidin/biotin (Vector Laboratories Inc., Burlingame, CA, USA) blocking step was performed prior to the first primary antibody, and sections were rinsed two times with 5\% NGS. To prevent cross reactivity and non-specific binding during double and triple fluorescence staining mouse IgG Fab Fragment (1/10, Jackson Labs, West Grove PA, USA) block for 1-2 hours was included after the addition of the first primary antibody. Primary antibodies were diluted in 5\% NGS at the following concentrations: 1:1000 guinea pig anti-porcine insulin (DAKO, Carpinteria, CA, USA), 1:5000 mouse monoclonal anti-glucagon (Sigma, Saint Louis, MO, USA), 1:1000 rabbit polyclonal to PDX1 (Abcam, Cambridge, MA, USA), 1:100 mouse anti-human CK19 (DAKO), 1:100 mouse anti-human vimentin (DAKO), 1:200 mouse anti-human/mouse NGN3 (DSHB, University of Iowa, Iowa City, IA, USA) and 1: 50 biotinylated anti-human SOX17 antibody (R\&D Systems, Minneapolis, MN, USA), and incubated for $1 \mathrm{~h}$, then washed two times with $5 \%$ NGS. The appropriate species- specific secondary antibody of goat anti guinea pig IgG Alexa Fluor 488, goat anti mouse IgG Alexa Fluor 488, goat anti rabbit IgG Alexa Fluor 594, or Streptavidin Alexa Fluor 594 were diluted 1:200 in 5\% NGS, and goat anti mouse IgG Alexa Fluor 350 was diluted 1:100 in 5\% NGS (Invitrogen Molecular Probes, Eugene, OR, USA) and applied for 30 minutes in the dark. Prior to application of second and following third rounds of primary antibody application, sections were washed twice in 5\% NGS. Negative controls were sections from each age point incubated without primary antibodies; all control slides yielded no labeling. Positive controls were adult human pancreatic sections. Slides were coverslipped with ProLong Gold anti-fade reagent with DAPI to counter stain nuclei or ProLong Gold antifade reagent without DAPI in the case of an AlexaFluor 350 secondary (Invitrogen) to preserve fluorescence. All slides were visualized with a Zeiss Axioscope II equipped with AxioCam MRc camera and analyzed with Axiovision 4.6 (Carl Zeiss MicroImaging GmbH, Göttingen, Germany).

\section{Image analysis}

Images collected from the fetal human pancreatic sections were separated into the three trimesters of human development. The following groupings were made: first trimester (1-12 wpc, $n=5)$, second trimester (13-24 wpc, $\mathrm{n}=14$ ), and third trimester (24- $38 \mathrm{wpc}$, $\mathrm{n}=5$ ) [27]. Furthermore, a $10 \mathrm{wpn}$ and an adult sample were grouped with the third trimester results providing a representation of a mature pancreas. To quantify the number of cells producing insulin, glucagon, or both insulin and glucagon, representative images $(n=2-3$ from each pancreatic section) were manually counted using ImageJ software [33]. Data is expressed as the percentage of insulin or glucagon expressing cells as a percentage of the total number of insulin and/or glucagon expressing cells.

\section{Statistical analysis}

Quantification Data Is Expressed As Mean \pm Sem. Statistical Comparisons Were Performed Using The Mann-Whitney Test, Assuming Representative Sampling And Independence. Given The Small Sample Size, This Test Is Used Since It Does Not Assume A Normal Distribution. The Acceptable Level Of Significance Was Considered $\mathrm{P}<0.05$.

\section{Results}

\section{Analysis of insulin and glucagon expression}

To characterize endocrine hormone expression and islet morphology during human pancreatic development we performed a qualitative immunohistochemical analysis of insulin and glucagon expression in fetal pancreas between 7.7 and $38 \mathrm{wpc}, 10 \mathrm{wpn}$, and in the adult pancreas as well as a quantitative analysis of the proportion of insulin and/or glucagon expression as a percent of total insulin and/or glucagon expressing cells. During the fetal period of human development, evolving islet morphology is observed from the initiation of endocrine hormone expression in scattered cells, to the clustering of those cells into islet-like structures, and further remodeling of those structures to acquire the adult islet phenotype.

Insulin and glucagon expression was absent at the beginning of the fetal period ( $7.7 \mathrm{wpc}$, not shown). At $8.4 \mathrm{wpc}$, insulin and glucagon expression was observed in the form of isolated single cells. Insulin and glucagon expression increased from $8.6 \mathrm{wpc}$ and the first islet like clusters were observed at $10.6 \mathrm{wpc}$ (Figure 1). These clusters consisted mostly of insulin positive cells with a few cells also co-expressing glucagon at the periphery of the positive structures (Figure 1). In addition, the size of 
Citation: Anderson SJ, Seeberger KL, Ellis CE, Eshpetzer A, Korbutt GS (2013) Immunohistochemical Characterization of Insulin, Glucagon, PDX1, SOX17 and NGN3 Expression in Human Fetal Pancreatic Development. J Stem Cell Res Ther 3: 148. doi:10.4172/2157-7633.1000148

Page 3 of 10

the islet structures increased at $12.1 \mathrm{wpc}$. To examine the composition of the insulin- and glucagon-expressing populations, we expressed their frequency relative to the total population of cells producing insulin and/or glucagon (Figure 2). Throughout the first trimester the percentage of insulin expressing cells $(36.4 \pm 6.3 \%)$ was higher than those expressing glucagon $(11.3 \pm 2.2 \%$, $\mathrm{p}<0.025)$, whereas there were $52.3 \pm 7.6 \%$ insulin/glucagon positive cells.

During the second trimester, the percentage of glucagon expressing cells $(59.7 \pm 2.5 \%)$ was greater than those expressing insulin $(25.58 \pm$ $1.5 \%, \mathrm{p}<0.25)$ and, by $15 \mathrm{wpc}$, the islet cluster size increased (Figure 1 ). The islet morphology in the second trimester was similar to rodent islets [29]. During the second trimester, a few co-expressing insulin and glucagon cells $(14.7 \pm 3.9 \%)$ of hormone expressing cells were located in the margin between the self-aggregated endocrine cells (Figure 1), in addition, amongst these islets there were smaller islet clusters similar to those of the first trimester with higher levels of co-expressing insulin and glucagon. The heterogeneous nature of islet morphology in the second trimester is indicative of a transitioning morphology into a more mature form.
In the third trimester (Figure 3), the percentage of glucagon expressing cells $(44.5 \pm 5.9 \%)$ is equivalent to insulin hormone expression $(53.7 \pm 5.2 \%, \mathrm{p}>0.05)$ and the islet morphology is similar to the adult human pancreas. Insulin and glucagon expressing cells become intermixed within the islet structures, and islet size becomes more homogeneous across the tissue sample. The islet morphology at 10 wpn is similar to other samples of the third trimester as well as that of the adult human islet demonstrating that mature islet morphology is acquired during the third trimester.

Co-expression of insulin and glucagon was observed from $8.4 \mathrm{wpc}$ up to and including $23 \mathrm{wpc}$ ( $23 \mathrm{wpc}$ data not shown). As a percentage of total endocrine cells (Figure 2), the greatest amount of cells co-expressing of insulin and glucagon is observed during the first trimester $(52.23 \pm 7.6)$ compared to the second $(14.7 \pm 3.9 \%, \mathrm{p}<0.025)$ and third $(1.88 \pm 0.68 \%, \mathrm{p}<0.025)$ trimesters, and post natal $(1.22 \pm$ $0.12 \%, \mathrm{p}<0.025)$. In the first trimester, most glucagon cells co-expressed insulin while cells only positive for insulin were located at the center of the islet-like clusters (Figure 1). Negligible co-expression of insulin

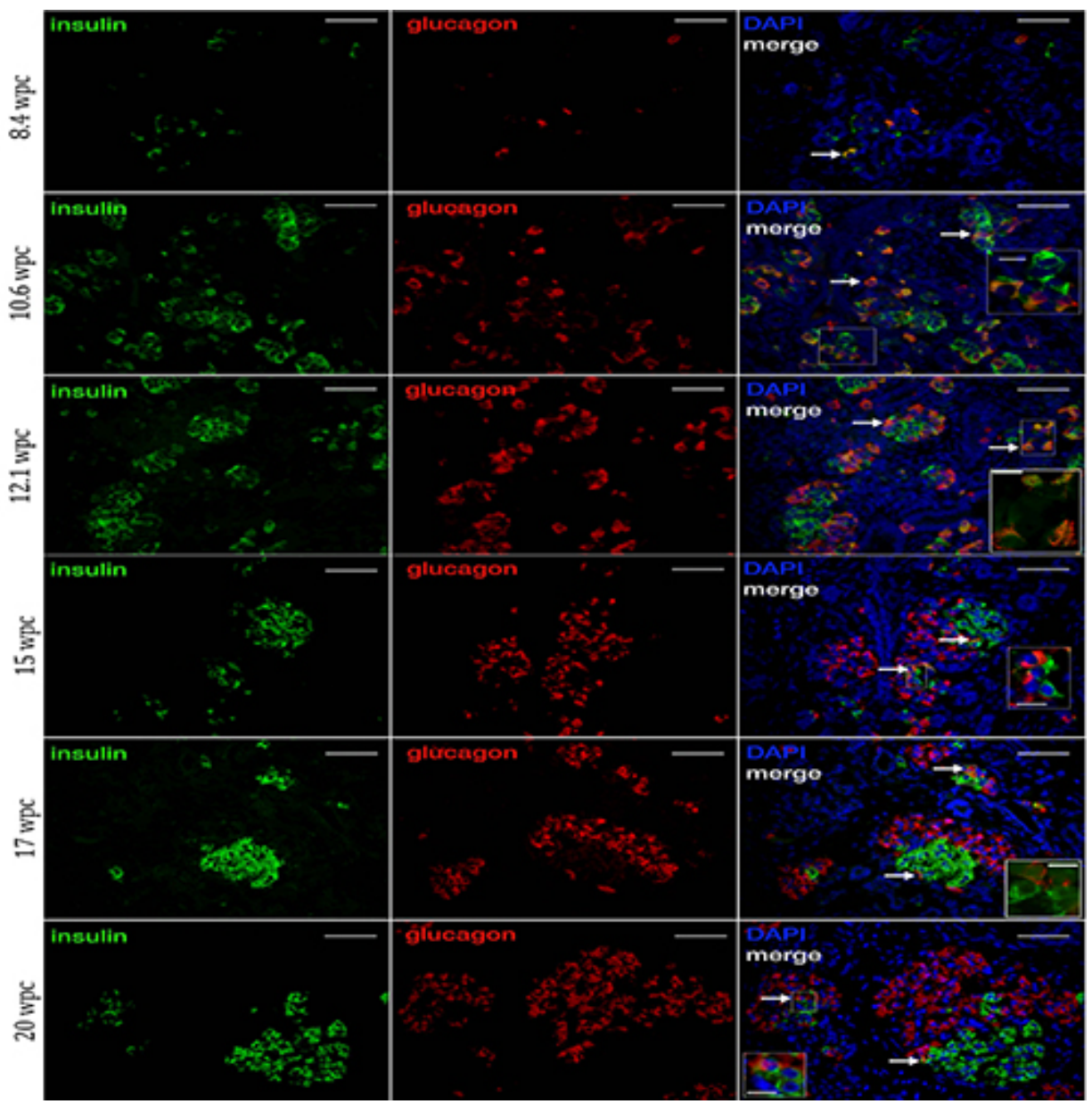

Figure 1: Insulin (green) and glucagon (red) expression during the first and second trimester of human fetal development (1- 24 wpc), as demonstrated by samples 8.4 , $10.6,12.1,15,17$, and 20 wpc. Arrows demonstrate examples of coexpressing insulin and glucagon cells. Prior to 8.4 weeks there is no positive insulin or glucagon staining. At $8.4 \mathrm{wpc}$ insulin and glucagon expression is observed in scattered cells. By $10.6 \mathrm{wpc}$ insulin and glucagon expression has significantly increased, and the first islet-like clusters are visible, consisting mostly of insulin positive cells, while a few insulin and glucagon co-expressing cells are observed on the periphery of the clusters. Islet size continues to increase between 10.6 and $12.1 \mathrm{wpc}$. During the second trimester, islet size continues to increase and glucagon expression is greater than insulin expression. In the second trimester, the majority of insulin and glucagon expressing cells are localized to islet structures rather than as isolated cells and self-aggregate. DAPI nuclear counterstain (blue). Areas denoted by a box are shown at higher magnificiation (inset). Higher magnification images of 12.1 and 17 wpc are shown without DAPI. Scale bars are 50 and $10 \mu \mathrm{M}$ respectively. 
Citation: Anderson SJ, Seeberger KL, Ellis CE, Eshpetzer A, Korbutt GS (2013) Immunohistochemical Characterization of Insulin, Glucagon, PDX1, SOX17 and NGN3 Expression in Human Fetal Pancreatic Development. J Stem Cell Res Ther 3: 148. doi:10.4172/2157-7633.1000148

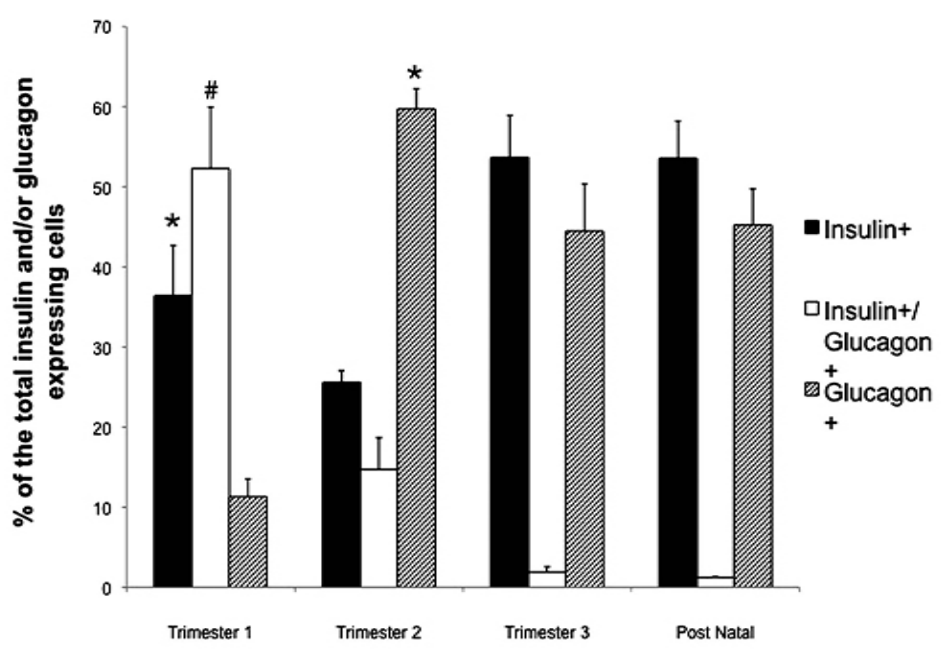

Figure 2: Quantification of insulin and glucagon expressing cells in the fetal and post natal pancreas. Percentage of total insulin expressing (black), co-expressing insulin and glucagon (white), and total glucagon expressing cells (cross hatch) relative to the total population of cells producing insulin and/or glucagon. Data is expressed mean \pm SEM, ${ }^{*}<<0.025$ within the same trimester and ${ }^{*} p<0.025$ for co-expression vs. other time points.

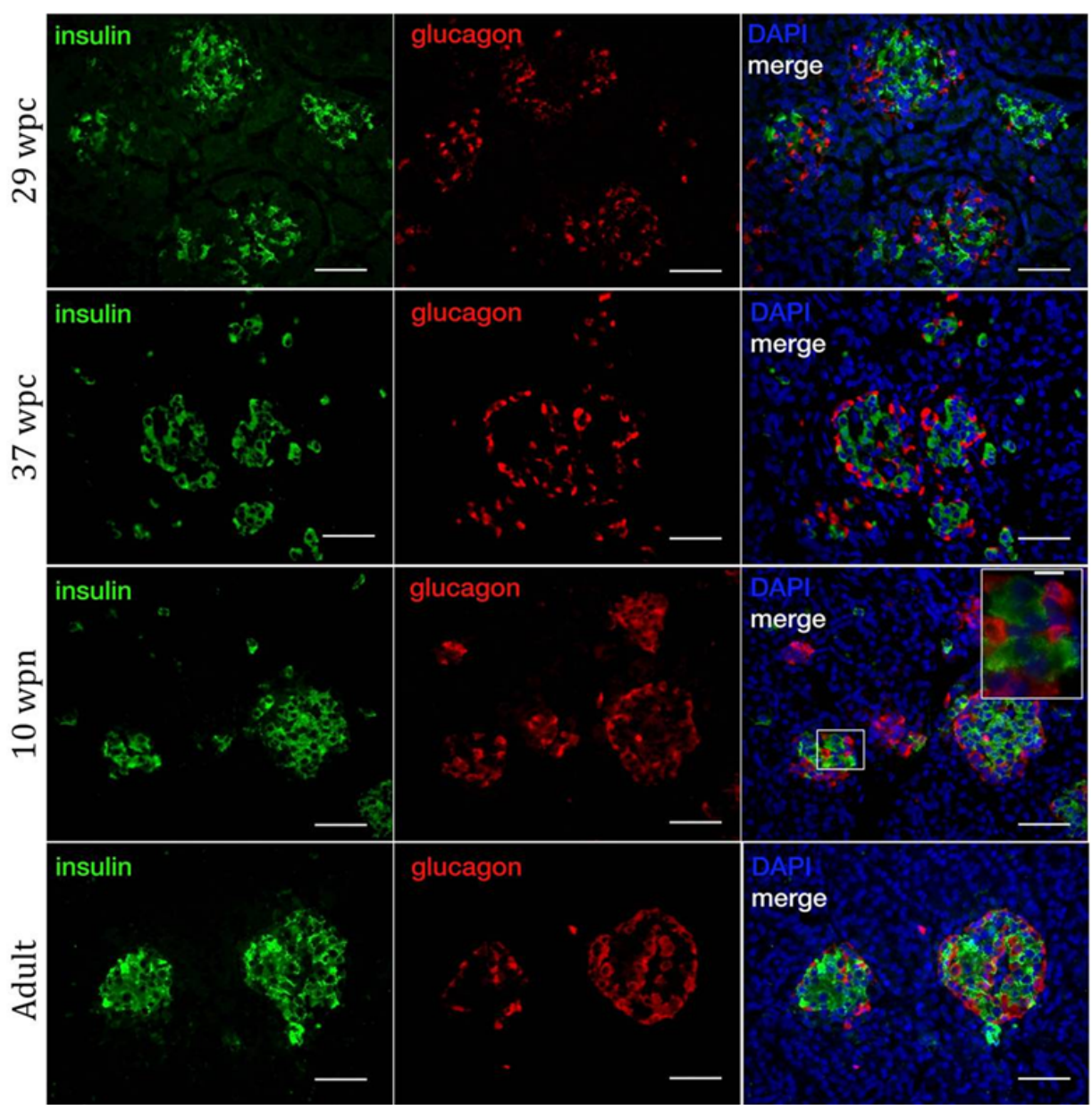

Figure 3: Insulin (green) and glucagon (red) expression during the third trimester of human fetal development (24 wpc-birth(38 wpc)) as demonstrated by samples 29 and $37 \mathrm{wpc}$, as well as $10 \mathrm{wpn}$, and adult. In the third trimester, the proportion of insulin and glucagon expressing cells are equivalent. Similar to the post natal samples, insulin and glucagon expressing cells are intermixed within the islets. In the third trimester and post natal samples, islet size is more homogeneous across the tissue section. No co-expression of insulin and glucagon was observed. DAPI nuclear counterstain (blue). Area denoted by a box is shown at higher magnification (inset). Scale bars are 50 and $10 \mu \mathrm{M}$ respectively. 
Citation: Anderson SJ, Seeberger KL, Ellis CE, Eshpetzer A, Korbutt GS (2013) Immunohistochemical Characterization of Insulin, Glucagon, PDX1, SOX17 and NGN3 Expression in Human Fetal Pancreatic Development. J Stem Cell Res Ther 3: 148. doi:10.4172/2157-7633.1000148

Page 5 of 10

and glucagon is observed amongst hormone positive cells in the third trimester and post natal samples (Figure 3).

\section{Analysis of PDX1 and insulin expressions}

In the first trimester (Figure 4), PDX1 expression is located in numerous duct-like structures, and is also co-expressed in all insulin cells. At 8.4 wpc scattered insulin expressing cells were located amongst the PDX1 positive cells. In the second trimester (Figure 4) a transition of PDX1 expression can be distinctly observed. Between 14.4 and 15 wpc (14.4 wpc data not shown), as islets increase in size and PDX1 association with the ductal structures is diminished, and increased in the insulin expressing cell clusters. In addition to PDX1 expression in all insulin positive cells, PDX1 is most pronounced in cells surrounding the insulin expressing cells. As maturation proceeds into the third trimester (Figure 5), PDX1 is minimally detected in the duct-like structures and decreased in those cells bordering the insulin positive cells. The first non-PDX1staining insulin cells are observed in the $37 \mathrm{wpc}$ sample. The late third trimester expression pattern resembled that of the adult pattern where PDX1 has less co-expression in the insulin positive cells and there are still a few expressing PDX1 cells at the periphery of the insulin expressing cells of the islets. As such it is assumed that islets are approaching maturity near the end of the fetal period in the third trimester.

\section{Expression of SOX17 and its co-localization with insulin and glucagon expression}

In the first trimester (Figure 6) SOX17 only expressing cells are initially scattered non-specifically amongst the insulin and glucagon expressing cells at 8.4 and $10.6 \mathrm{wpc}$. Occasional SOX17 and insulin coexpressing cells were observed in the 8.4 through $14.4 \mathrm{wpc}$ samples. From 13 through 29 wpc (Figures 6 and 7), the SOX17 expressing cells are spatially associated with the glucagon expressing cells and emphasized in the second trimester samples, where the insulin and glucagon cells have self-aggregated, and the SOX17 expressing cells are found in the glucagon cell clusters. Between 29 and $38 \mathrm{wpc}$ in the third trimester (Figure 7), the insulin, glucagon, and SOX17 expressing cells disperse amongst the entire islet to acquire the adult phenotype.

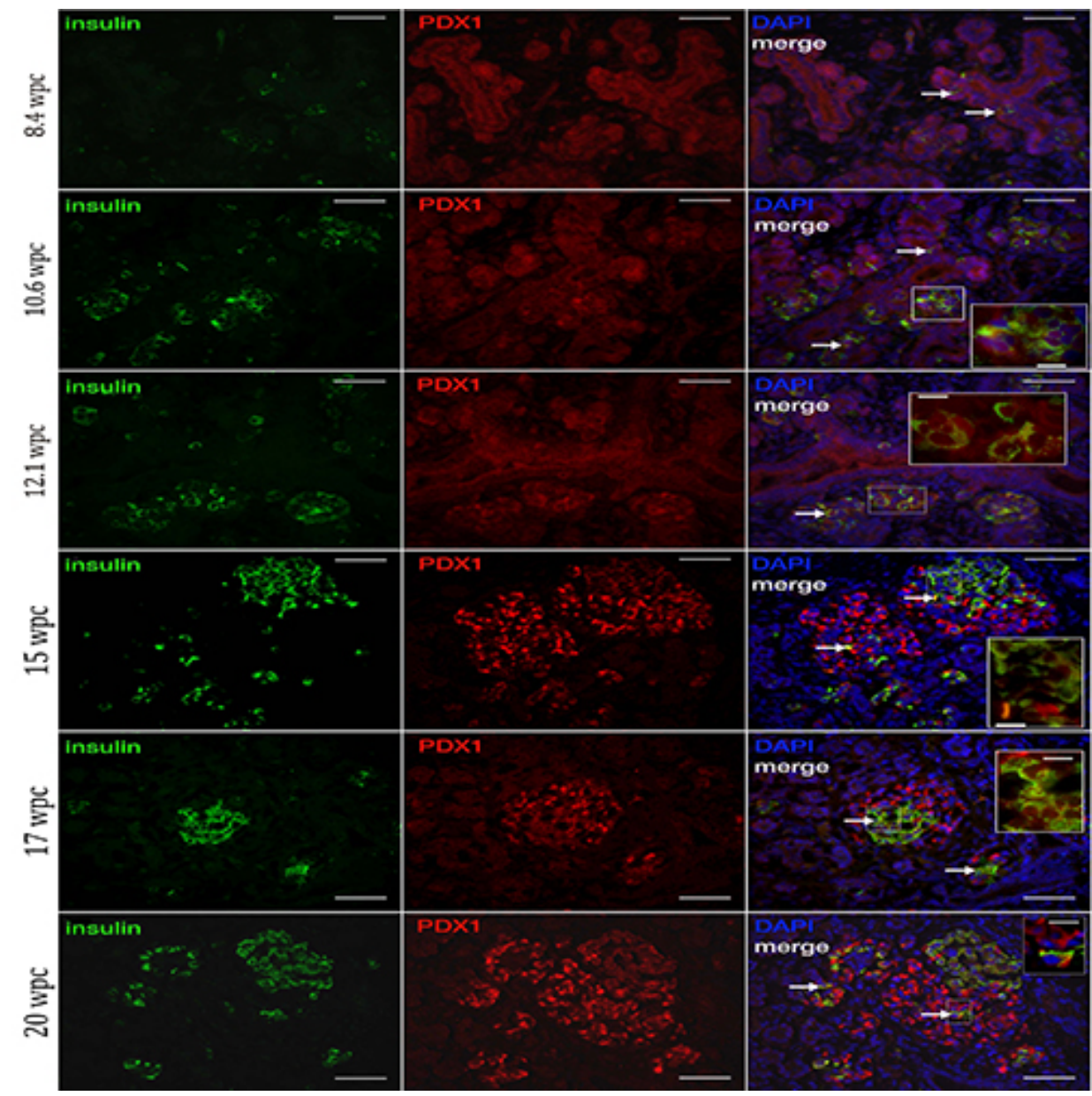

Figure 4: Insulin (green) and PDX1 (red) expression during the first and second trimester of human fetal development (1-24 wpc), as demonstrated by samples 8.4, 10.6, $12.1,15,17$, and $20 \mathrm{wpc}$. Arrows demonstrate examples of co-expressing insulin and PDX1 cells. In the first trimester, PDX1 expressing cells are located in numerous duct-like structures, and insulin expressing cells co-express PDX1. At $8.4 \mathrm{wpc}$, scattered insulin expressing cells are located amongst the duct-like PDX1 expressing cells. As more insulin expressing cells aggregate at 10.6 and $12.1 \mathrm{wpc}$, the islet-like structures seem to bud off from the PDX1 expressing duct-like structures. In the second trimester, PDX1 expression is localized mainly to islet structures, and PDX1 expression is most pronounced in cells surrounding the insulin expressing cells. All insulin expressing cells co-express PDX1. The islet structures have less spatial association to the duct-like PDX1 expressing structures. DAPI nuclear counterstain (blue). Areas denoted by a box are shown at higher magnificiation (inset). Higher magnification images of $12.1,15$ and 17 wpc are shown without DAPI. Scale bars are 50 and $10 \mu \mathrm{M}$ respectively. 
Citation: Anderson SJ, Seeberger KL, Ellis CE, Eshpetzer A, Korbutt GS (2013) Immunohistochemical Characterization of Insulin, Glucagon, PDX1, SOX17 and NGN3 Expression in Human Fetal Pancreatic Development. J Stem Cell Res Ther 3: 148. doi:10.4172/2157-7633.1000148

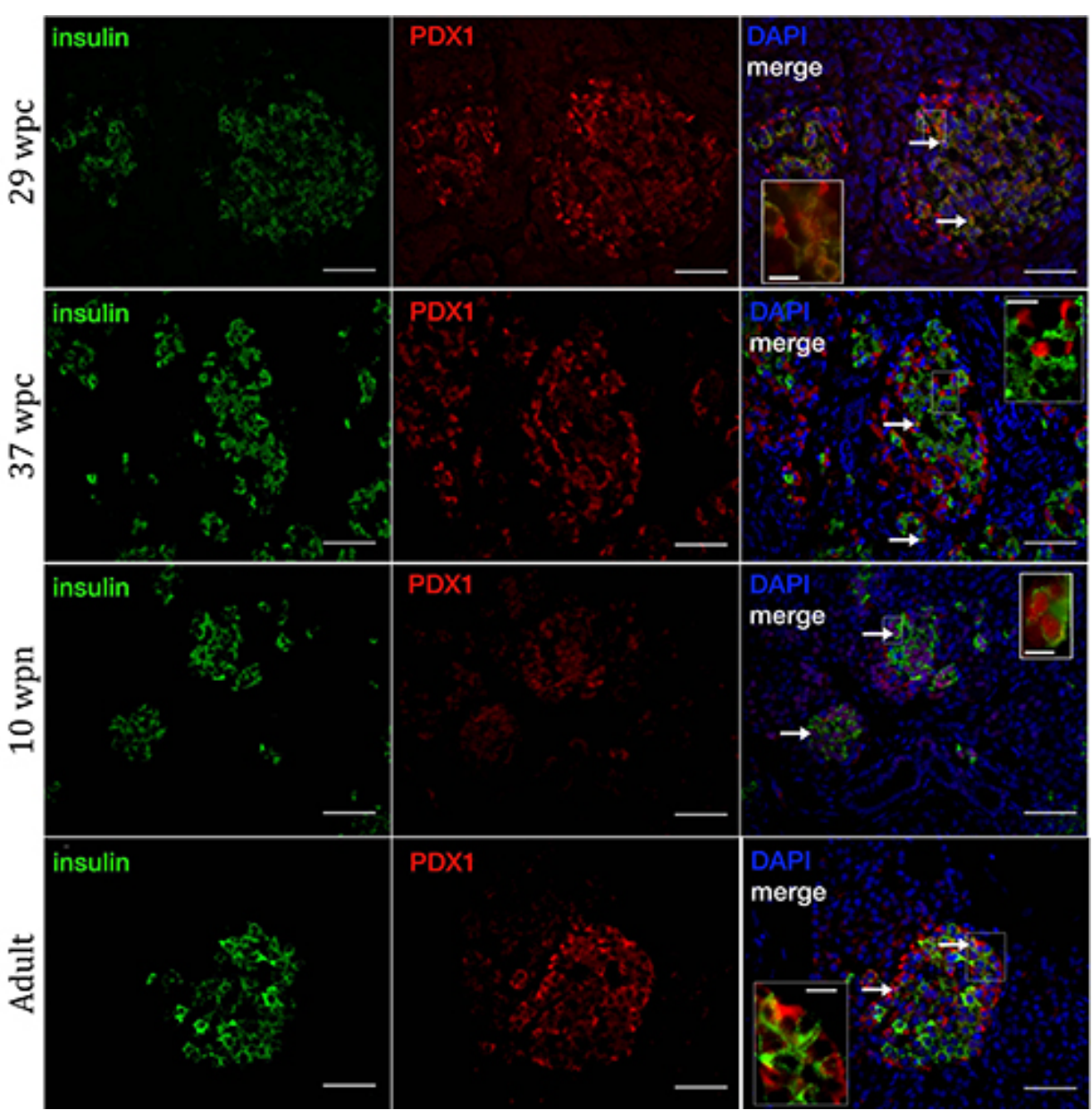

Figure 5: Insulin (green) and PDX1 (red) expression during the third trimester of human fetal development (24wpc-birth (38wpc)) as demonstrated by samples 29 and 37 wpc, as well as $10 \mathrm{wpn}$, and adult. Arrows demonstrate examples of coexpressing insulin and PDX1 cells. In the third trimester, PDX1 expression is minimally detected in duct-like structures, and expression is also proportionatally less compared to the second trimester in the islet structures. The third trimester expression pattern is similar to the post natal and adult, where less PDX1 is co-expressed in insulin cells, and a few PDX1 alone cells are located at the periphery of the insulin cells. The first insulin alone expressing cell is observed at 37 wpc. DAPI nuclear counterstain (blue). Areas denoted by a box are shown at higher magification (inset) without DAPI. Scale bars are 50 and $10 \mu \mathrm{M}$.

\section{Analysis of NGN3 expression}

NGN3 positive cells were observed in the first trimester up until 14.4 wpc and at 10 wpn but absent in the second and third trimesters (15$38 \mathrm{wpc}$ ) as well as the adult pancreas (not shown). At $7.7 \mathrm{wpc}$, NGN3 cells were located at the edge of a lumen (Figure 8). At this time point the entire developing pancreas stained positive for vimentin indicating mesenchymal tissue and all NGN3 positive cells co-expressed vimentin (Figure 8). Neither CK19 cells indicating ductal epithelium nor insulin positive cells were detected at $7.7 \mathrm{wpc}$ (not shown). At $8.4 \mathrm{wpc}$ when scattered insulin positive cells are apparent within the mesenchyme, the ductal epithelium has formed and CK19 positive cells line these early ducts that are branched (Figure 8). NGN3 positive cells that co-express vimentin are in elongated cell clusters (Figure 9) and scattered in the mesenchyme around the CK19 positive ductal tree (Figure 8). At 10.6 wpc, NGN3 positive cells are concentrated around the forming isletlike cell clusters (Figure 9). The CK19 positive ducts condense and less branching of CK19 positive cells is observed (not shown). The number of NGN3 positive cells peak between 10.6-12.1 wpc, and as the islet and duct structures form the number vimentin positive cells decline. At 12.1 and $14.4 \mathrm{wpc}$, the numbers of vimentin positive cells have decreased (Figure 8), the ductal epithelium resembles mature ducts (Figure 8 ) as seen in the adult and NGN3 positive cells still surround the developing islet and also infiltrate the islet at this time point (Figure 9). Less NGN3 positive cells are observed at 14.4 and are undetectable past $15 \mathrm{wpc}$ (not shown). Post natal (10 wpn), a few NGN3 positive cells are detected scattered throughout the pancreas (Figure 8) and within the islet (Figure 9). NGN3 positive cells co-expressed vimentin throughout fetal pancreas development and did not co-express insulin or CK19.

\section{Discussion}

There are significant differences between human and rodent biology, islet composition, and development [4-12]. For instance, the timing of corresponding initial insulin expression is not equivalent; mice begin expressing insulin positive cells at E11, projected to human development, expression would be expected at 33 days post conception (4.7 wpc) [5]. However, in this study we did not observe insulin positive cells until $8.4 \mathrm{wpc}$ (Figure 1). Morphological studies indicate that mouse islets are properly formed within a few days of birth following a secondary wave of $\beta$-cell differentiation [7] where as human islets 
Citation: Anderson SJ, Seeberger KL, Ellis CE, Eshpetzer A, Korbutt GS (2013) Immunohistochemical Characterization of Insulin, Glucagon, PDX1, SOX17 and NGN3 Expression in Human Fetal Pancreatic Development. J Stem Cell Res Ther 3: 148. doi:10.4172/2157-7633.1000148

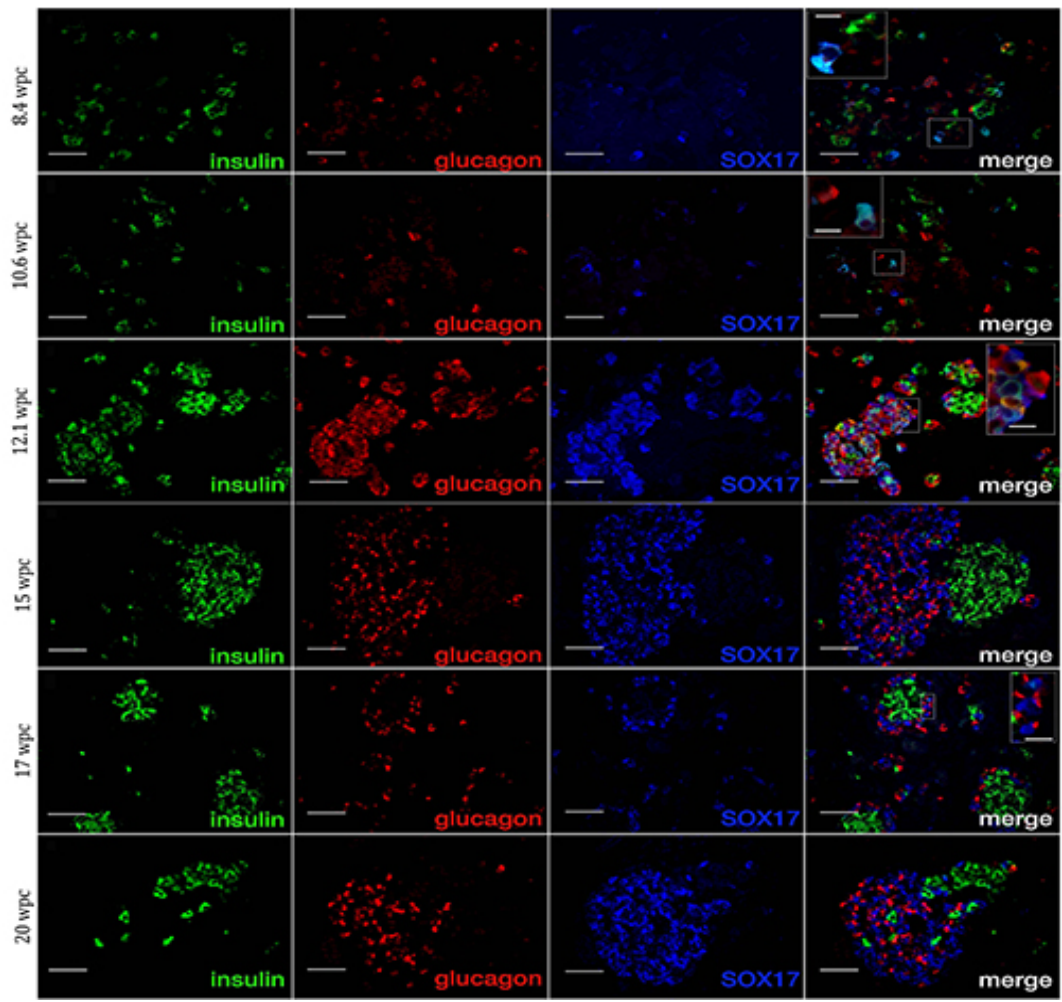

Figure 6: Insulin (green), glucagon (red) and SOX17 (blue) expression during the first and second trimester of human fetal development (1- 24 wpc), as demonstrated by samples $8.4,10.6,12.1,15,17$, and 20 wpc. In the first trimester, SOX17 expressing cells are scattered non-specifically amongst the insulin and glucagon expressing cells. Upon islet-like cluster formation at 10.6 wpc SOX17 expressing cells are also found in the islet-like clusters. SOX17 is not co-expressed in glucagon expressing cells, however select insulin expressing cells appear to co-express SOX17. In the second trimester, SOX17 expressing cells are spatially associated with glucagon expressing cells within the islet structures. At 15, 17, and 20 wpc SOX17 is not coexpressed in insulin or glucagon expressing cells. Areas surrounded by a box are shown at higher magnification (inset). Scale bars are 50 and $10 \mu \mathrm{M}$ respectively.

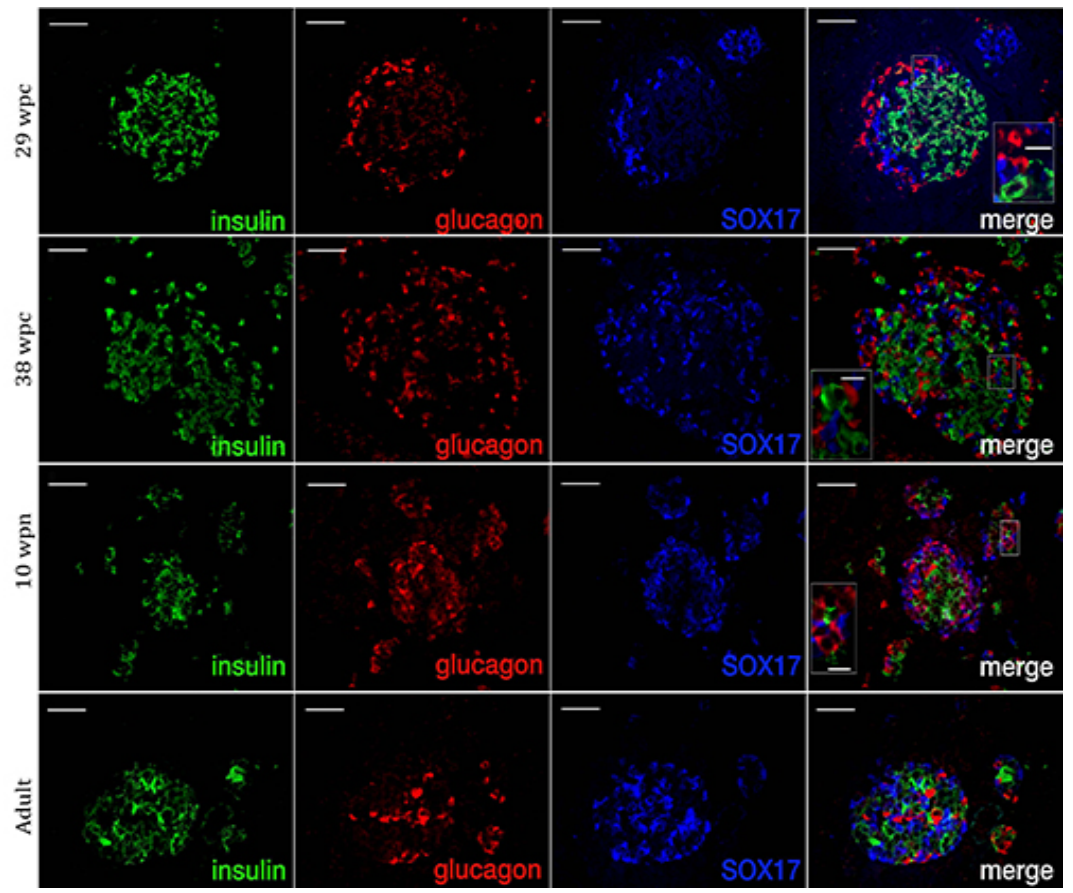

Figure 7: Insulin (green), glucagon (red) and SOX17 (blue) expression during the third trimester of human fetal development (24wpc-birth(38wpc)) as demonstrated by samples 29 and $38 \mathrm{wpc}$, as well as $10 \mathrm{wpn}$, and adult. In the third trimester, SOX17 expressing cells are initially associated with glucagon expressing cells at $29 \mathrm{wpc}$, however as the insulin and glucagon expressing cells become intermixed by 37 wpc and post natally, the SOX17 expressing cells also are intermixed with other cell types within the islets. SOX17 is not co-expressed in insulin or glucagon expressing cells in the third trimester or post natally. Areas surrounded by a box are shown at higher magnification (inset). Scale bars are 50 and $10 \mu \mathrm{M}$ respectively. 
Citation: Anderson SJ, Seeberger KL, Ellis CE, Eshpetzer A, Korbutt GS (2013) Immunohistochemical Characterization of Insulin, Glucagon, PDX1, SOX17 and NGN3 Expression in Human Fetal Pancreatic Development. J Stem Cell Res Ther 3: 148. doi:10.4172/2157-7633.1000148

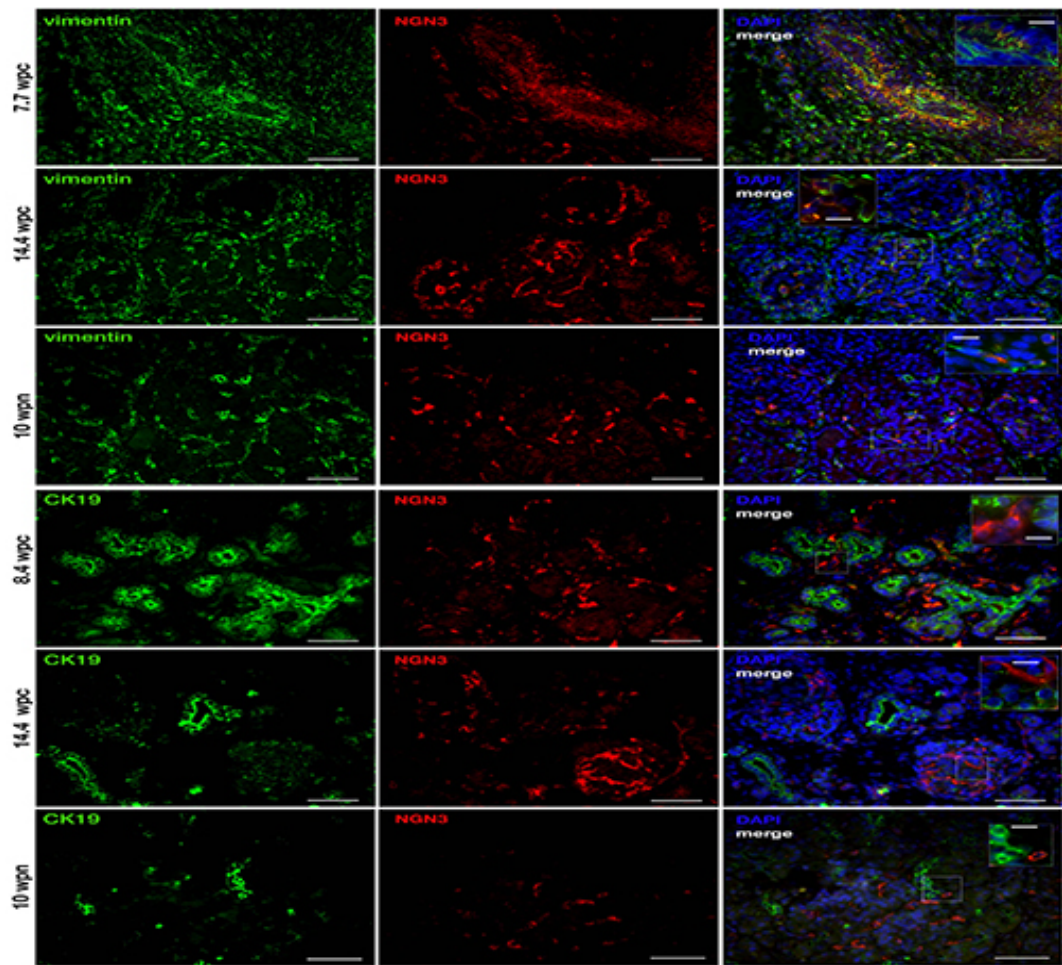

Figure 8: Vimentin (green), CK19 (green), and NGN3 (red) expression during the first trimester $(7.7,8.4,14.4$ ) and post natal (10 wpn). At 7.7 wpc the pancreas is composed of vimentin positive cells (mesenchyme). NGN3 positive cells are lining a lumen and all the NGN3 positive cells co-express vimentin (inset). At 14.4 wpc, there are less vimentin positive cells and the NGN3 positive cells surround cell clusters (forming islets). All the NGN3 positive cells co-express vimentin and are also visible within the islet structure. NGN3 cells at 10 wpn are scattered throughout the pancreas and within the islet, these cells co-express vimentin. At 8.4 wpc the ductal tree that has formed is comprised of CK19 positive cells. NGN3 positive cells are scattered in and amongst the spaces around the ductal tree. At 14.4 wpc as the ducts are condensing and mature ducts are forming, the NGN3 positive cells are closely associated with the forming islets and are found around and within the forming islet. At 10 wpn the NGN3 positive cells are scattered throughout the section and do not appear to be associated with the mature ducts (CK19). Areas denoted by a box are shown at higher magnification (inset). Scale bars are 50 and $10 \mu \mathrm{M}$ respectively.

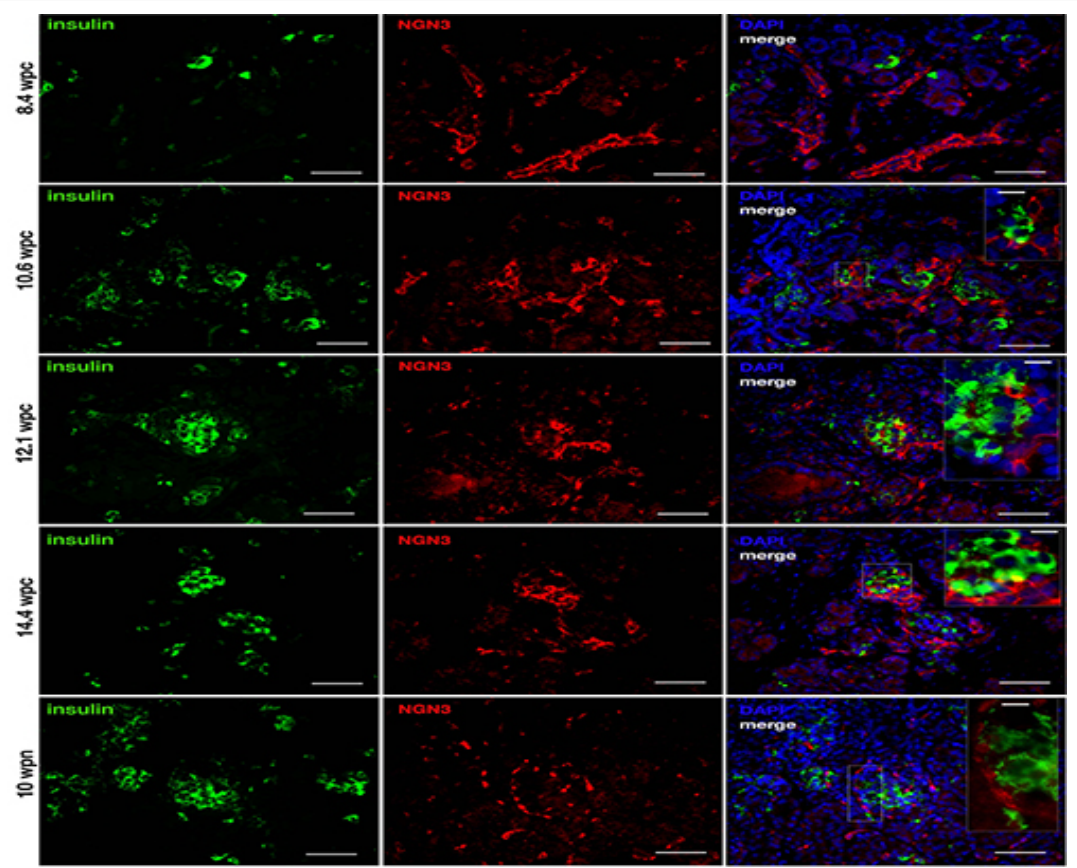

Figure 9: Insulin (green) and neurogenin3 (NGN3, red) expression during the first trimester (8.4, 10.6, 12.1, 14.4) and post natal (10 wpn). At 8.4 wpc NGN3 cells are filamentous, elongated and are scattered throughout the section and arranged in branch-like formations. The NGN3 positive cells are not associated with the scattered insulin positive cells. At $10.6 \mathrm{wpc}$, NGN3 cells surround the first islet-like cell clusters in a wave-like pattern and the number of NGN3 positive cells appears to peak at this time point. At 12.1 and $14.4 \mathrm{wpc}$, while the NGN3 cell number decreases these cells are more closely associated with the forming islets and are found around and within the forming islet. Post natal (10 wpn), a few NGN3 cells are evident near and in the islet as well as scattered throughout the pancreas section. There is no evidence of insulin and NGN3 co-expression at any of these time points. Areas denoted by a box are shown at higher magnification (inset). Scale bars are 50 and $10 \mu \mathrm{M}$ respectively. 
initially appear in the first trimester and attain a mature form during the third trimester (Figures 1 and 3). Several studies have demonstrated that human endocrine pancreas formation is unlike the mouse as there is no evidence of a primary [26] or secondary transition [15] in human endocrine pancreas development.

As studies using human fetal pancreatic tissue are relatively new in the field of developmental research, the timing and developmental staging of the human fetal pancreas amongst studies is not always consistent [14,15,21,26,33]. For instance, several studies observe endocrine hormone expression as early as $9 \mathrm{wpc}[4,14,19]$, while others have noted a few insulin positive cells at $6 \mathrm{wpc}$ [4] and we observed that insulin and glucagon expression initiates between 7.4 and 8.4 wpc. By assessing human embryonic tissue as early as $22-26$ days postconception (dpc) Jennings et al. [26] noted infrequent insulin positive cells as early as $47-52 \mathrm{dpc}$ which is close to $7 \mathrm{wpc}$. Early endocrine cells that co-express both insulin and glucagon that later mature to mono-hormonal cell $(17,5,20)$ were also observed in our study from 8.4-23 wpc however, Sarker et al. [15] observed only single hormone cells through 9-23 wpc. These observations can be attributed to the limited availability of human fetal pancreatic tissue for study thus, the advantage of our study was that we were able to observe the entire period of fetal development and visualize islet development from the initiation of endocrine expression through to the formation of mature islets. Our observations are consistent with Bocian-Sobkowska et al. [14] who described the four stages of human islet development: first, scattered polyhormonal (9-10 wpc), second, immature polyhormonal islet stage (11-15 wpc), third, insulin monohormonal core islet stage (16-29 wpc) and finally polymorphic islet stage (30 wpc onward).

In transcription factor studies of the 8 to $21 \mathrm{wpc}$ fetal pancreatic samples Lyttle et al. [21] noted initial PDX1 expression in CK19 positive cells, following this the PDX1 and CK19 co-expression decreased with a simultaneous increase in PDX1 and insulin coexpressing cells. According to Piper et al. [5] in early fetal development, CK19 marks pancreatic epithelial cells. In our study, we also note that PDX1 expression is initially localized to duct-like structures, and later in development it shifts to be islet associated.

SOX17 is expressed early in murine endoderm development and specifies cell lineage (22-27). Recently Wang et al. [25] and Fishman et al. [27] utilizing similar lineage tracing strategies revealed, by isolating SOX17 expressing human embryonic stem cells that human gastrointestinal lineages including the pancreas are derived from SOX17 positive cells. Jennings et al. [26] when assessing post implantation embryos observed SOX17 at 25-27 dpc prior to PDX1 expression in the foregut of the developing human embryo. Though their study extended to $10 \mathrm{wpc}$, SOX17 was not detected in later stages of the duodenum or pancreas past $30-33 \mathrm{dpc}$. Our observations revealed that SOX17 is present during early islet formation (8.4-14.4 $\mathrm{wpc}$ ) and is closely associated with glucagon cells during the second trimester. However, in the third trimester SOX17 cells dispersed among the islet field. Together, this may suggest that SOX17 may exhibit a temporal expression pattern where it is expressed early in embryonic development, then disappears and is later re-expressed commencing around $8 \mathrm{wpc}$.

There have been extensive reports detailing the pattern of NGN3 expression during mouse endocrine pancreas development [28-32]. During the temporal development of the mouse pancreas NGN3 is expressed during the primary and secondary transitions, peaking during the secondary transition and is diminished at the tertiary transition and post natal [34]. This pattern has been described as biphasic expression [35]. To date there is no evidence to suggest that these transitions occur during human endocrine pancreas development or that NGN3 is temporally expressed $[15,26]$. However, reports describing NGN3 expression during human endocrine pancreas development are inconsistent.

Our NGN3 observations were unique. At $7.7 \mathrm{wpc}$ when insulin, glucagon and CK19 cells are not yet detectable and the pancreas consists entirely of mesenchyme NGN3 cells that co-expressed vimentin were observed surrounding a lumen (Figure 8). The NGN3 cells remained in the mesenchyme that surrounded the developing epithelium and endocrine tissue (Figures 8 and 9). At 10.6-12.1 wpc NGN3 cells peaked and were closely associated with the forming islets. By 12.1 and 14.4 in addition to surrounding new islets NGN3 cells infiltrated the islet. NGN3 was not detected in sections 15-38 wpc but did reappear post natal as cells scattered throughout the pancreas and in the islet. Similar findings have been reported. Sarker et al. [15] described NGN3 positive cells scattered among epithelial clusters and detected NGN3 in 9-13 wpc, as well as mRNA for NGN3 up to 23 weeks. Hanley et al. [33] also reported no co-localising of insulin or PDX1 in NGN3 cells as did Jennings et al. [26]. Jennings et al. [26] did quantify and report a significant increase in NGN3 cells at 9-10 wpc that coincided with the increase of insulin cells. The lack of co-expression with endocrine hormones, PDX1 and ductal epithelium correlates with mouse studies where NGN3 is extinguished when hormone positive cells appear [28,30]. Only Lyttle et al. [21] have reported co-expression of NGN3 with insulin, glucagon and PDX1, and they were also able to detect NGN3 as late as 21 weeks.

Human developmental studies are inherently difficult due to the procurement of human fetal tissue. As such the numbers of samples per age point are limited. The main strength of this study lies in that we were able to obtain a reasonable number of samples across the entire fetal development period. This provided a better overview of the entire process of endocrine cell clustering to form mature islets. However, despite a limited pool of samples, the results attained do illustrate a progressive picture of human islet development. Overall, this study was able to present a unique qualitative assessment of insulin, glucagon, PDX1, SOX17 and NGN3 expression and co-expression patterns, during human fetal pancreatic development. Comprehensive morphologic changes during the formation and development of human fetal islets were observed especially with respect to SOX17 and NGN3. In combination with in vitro human embryonic stem cell isolation studies [24,25,27], in vivo characterization of $\beta$-cell progenitors during fetal development, will improve progenitor cell isolation and differentiation of viable $\beta$-cell progenitors destined for transplantation.

\section{Acknowledgements}

We thank the staff of Alberta Health Services, the ADI IsletCore (University of Alberta), and Edmonton Clinical Islet Transplant Program (Alberta Health Services) for providing pancreatic tissue sections. We thank Lynette Elder, Sheena Lesyk, Jessica Mark, and Bethany Ostrowerka, for technical assistance. The NGN3 antibody developed by Ole D Madsen was obtained from the Developmental Studies Hybridoma Bank developed under the auspices of the NICHD and maintained by The University of lowa, Department of Biology, lowa City IA 52242. Funding for this project was provided by the Canadian Institutes of Health Research (MOP8030).

\section{References}

1. Bernardo AS, Hay CW, Docherty K (2008) Pancreatic transcription factors and their role in the birth, life and survival of the pancreatic beta cell. Mol Cell Endocrinol 294: 1-9.

2. Wilson ME, Scheel D, German MS (2003) Gene expression cascades in pancreatic development. Mech Devel 120: 65-80. 
Citation: Anderson SJ, Seeberger KL, Ellis CE, Eshpetzer A, Korbutt GS (2013) Immunohistochemical Characterization of Insulin, Glucagon, PDX1, SOX17 and NGN3 Expression in Human Fetal Pancreatic Development. J Stem Cell Res Ther 3: 148. doi:10.4172/2157-7633.1000148

3. Servitja JM, Ferrer J (2004) Transcriptional networks controlling pancreatic development and beta cell function. Diabetologia 47: 597-613.

4. Jeon J, Correa-Medina M, Ricordi C, Edlund H, Diez JA (2009) Endocrine cell clustering during human pancreas development. J Histochem Cytochem 57 811-824.

5. Piper K, Brickwood S, Turnpenny LW, Cameron IT, Ball SG (2004) Beta cell differentiation during early human pancreas development. J Endocrinol 181: 11-23.

6. Richardson MK, Hanken J, Gooneratne ML, Pieau C, Raynaud A, et al. (1997) There is no highly conserved embryonic stage in the vertebrates: Implications for current theories of evolution and development. Anat Embryol (Berl) 196: 91-106.

7. Slack JM (1995) Developmental biology of the pancreas. Development 121 1569-1580.

8. Brissova M, Fowler MJ, Nicholson WE, Chu A, Hirshberg B, et al. (2005) Assessment of human pancreatic islet architecture and composition by laser scanning confocal microscopy. J Histochem Cytochem 53: 1087-1097.

9. Cabrera O, Berman DM, Kenyon NS, Ricordi C, Berggren PO, et al. (2006) The unique cytoarchitecture of human pancreatic islets has implications for islet cell function. Proc Natl Acad Sci USA 103: 2334-2339.

10. Bosco D, Armanet M, Morel P, Niclauss N, Sgroi A, et al. (2010) Unique arrangement of alpha- and beta-cells in human islets of langerhans. Diabetes 59: $1202-1210$.

11. Rodriguez-Diaz R, Abdulreda MH, Formoso AL, Gans I, Ricordi C, et al. (2011) Innervation patterns of autonomic axons in the human endocrine pancreas. Cell Metab 14: 45-54.

12. Shiao MS, Liao BY, Long M, Yu HT (2008) Adaptive evolution of the insulin two-gene system in mouse. Genetics 178: 1683-1691.

13. Stefan Y, Grasso S, Perrelet A, Orci L (1983) A quantitative immunofluorescent study of the endocrine cell populations in the developing human pancreas. Diabetes 32: 293-301.

14. Bocian-Sobkowska J, Zabel M, Wozniak W, Surdyk-Zasada J (1999) Polyhormonal aspect of the endocrine cells of the human fetal pancreas. Histochem Cell Biol 112: 147-153.

15. Sarkar SA, Kobberup S, Wong R, Lopez AD, Quayum N, et al. (2008) Global gene expression profiling and histochemical analysis of the developing human fetal pancreas. Diabetologia 51: 285-297.

16. Bouwens L, Lu WG, De Krijger R (1997) Proliferation and differentiation in the human fetal endocrine pancreas. Diabetologia 40: 398-404.

17. Polak M, Bouchareb-Banaei L, Scharfmann R, Czernichow P (2000) Early pattern of differentiation in the human pancreas. Diabetes 49: 225-232.

18. Meier JJ, Kohler CU, Alkhatib B, Sergi C, Junker T, et al. (2010) Beta-cell development and turnover during prenatal life in humans. Eur $\mathrm{J}$ Endocrino 162: 559-568.

19. Riedel MJ, Asadi A, Wang R, Ao Z, Warnock GL, et al. (2012) Immunohistochemical characterisation of cells co-producing insulin and glucagon in the developing human pancreas. Diabetologia 55: 372-381.

20. Kaneto H, Miyatsuka T, Shiraiwa T, Yamamoto K, Kato K, et al. (2007) Crucial role of PDX-1 in pancreas development, beta-cell differentiation, and induction of surrogate beta-cells. Curr Med Chem 14: 1745-1752.

21. Lyttle BM, Li J, Krishnamurthy M, Fellows F, Wheeler MB, et al. (2008) Transcription factor expression in the developing human fetal endocrine pancreas. Diabetologia 51: 1169-1180.

22. Wells JM, Melton DA (1999) Vertebrate endoderm development. Annual Rev Cell Devel Biol 15: 393-410.

23. Xu X, Kahan B, Forgianni A, Jing P, Jacobson L, et al. (2006) Endoderm and pancreatic islet lineage differentiation from human embryonic stem cells. Cloning Stem Cells 8: 96-107.
24. McDonald E, Krishnamurthy M, Goodyer CG, Wang R (2009) The emerging role of SOX transcription factors in pancreatic endocrine cell development and function. Stem Cells Dev 18: 1379-1388.

25. Wang P, Rodriguez RT, Wang J, Ghodasara A, Kim SK (2011) Targeting SOX17 in human embryonic stem cells creates unique strategies for isolating and analyzing developing endoderm. Cell Stem Cell 4: 335-346.

26. Jennings RE, Berry AA, Kirkwood-Wilson R, Roberts NA, Hearn T, et al. (2013) Development of the human pancreas from foregut to endocrine commitment Diabetes.

27. Fishman B, Segev H, Kopper O, Nissenbaum J, Schulman M, et al. (2012) Targeting pancreatic progenitor cells in human embryonic stem cell differentiation for the identification of novel cell surface markers. Stem Cell Rev 8: 792-802.

28. Scheiwtzgebel VM, Scheel DW, Conners JR, Kalamaras J, Lee JE, et al. (2000) Expression of neruogenin3 reveals an islet cell precurosor population in the pancreas. Development 127: 3522-3542.

29. Gu G, Dubauskaite J, Melton DA (2002) Direct evidence for the pancreatic lineage: NGN3+ cells are islet progenitors and are distinct from duct progenitors. Development 129: 2447-2257.

30. Gradwohl G, Dierich A, LeMeur M, Guillemot F (2000) Neurogenin3 is required for the development of the four endocrine cell lineages of the pancreas. Proc Nat Acad Sci 97: 1607-1611.

31. Grapin-Botton A, Jahithia AR, Melton DA (2001) Key events of pancreas formation are triggered in gut endoderm by ectopic expression of pancreatic regulatory genes. Genes Dev 15: 444-454.

32. Jensen J, Heller RS, Funder-Nielsen $T$, Pedersen EE, Lindsell $C$, et al. (2000) Independent development of pancreatic alpha- and beta-cells from neurogenin3-expressiong precursors: a role for the notch pathway in repression of premature differentiation. Diabetes 49: 153-176

33. Abramoff MD, Magalhaes PJ, Ram SJ (2004) Image processing with Image. J Biophotonics International 11: 36-42.

34. Hanley KP, Hearn T, Berry A, Carvell MJ, Patch AM, et al. (2010) In vitro expression of NGN3 identifies RAB3B as the predominant Ras-associated GTP-binding protein 3 family member in human islets. J Endocrinol 207: 151 161.

35. Johansson KA, Dursun U, Jordan N, Gu G, Beermann F, et al. (2007) Tempora control of neurogenin 3 activity in pancreas progenitors reveals competence windows for the generation of different endocrine cell types. Dev Cell 12: 457 465 